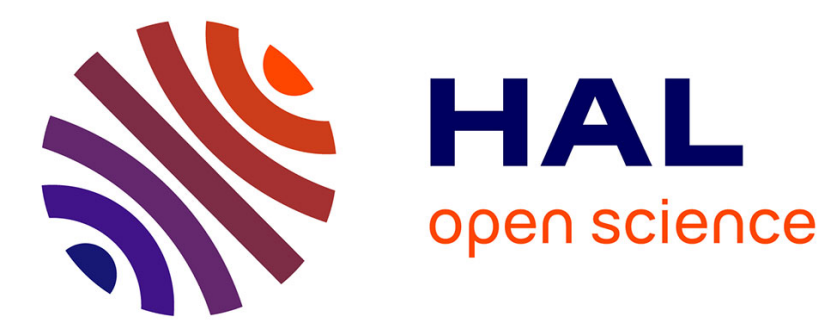

\title{
Optimal robust quantum control by inverse geometric optimization
}

\author{
Ghassen Dridi, Kaipeng Liu, Stéphane Guérin
}

\section{To cite this version:}

Ghassen Dridi, Kaipeng Liu, Stéphane Guérin. Optimal robust quantum control by inverse geometric optimization. Physical Review Letters, 2020, 125 (25), 10.1103/PhysRevLett.125.250403. hal-03253079

\section{HAL Id: hal-03253079 \\ https://hal.science/hal-03253079}

Submitted on 8 Jun 2021

HAL is a multi-disciplinary open access archive for the deposit and dissemination of scientific research documents, whether they are published or not. The documents may come from teaching and research institutions in France or abroad, or from public or private research centers.
L'archive ouverte pluridisciplinaire HAL, est destinée au dépôt et à la diffusion de documents scientifiques de niveau recherche, publiés ou non, émanant des établissements d'enseignement et de recherche français ou étrangers, des laboratoires publics ou privés. 


\title{
Optimal robust quantum control by inverse geometric optimization
}

\author{
Ghassen Dridi, ${ }^{1,2}$ Kaipeng Liu ${ }^{3}$ and Stéphane Guérin ${ }^{3, *}$ \\ ${ }^{1}$ Institut Supérieur des Sciences Appliquées et de Technologies de Gafsa, \\ Université de Gafsa, Campus Universitaire Sidi Ahmed Zarroug, Gafsa 2112, Tunisia \\ ${ }^{2}$ Laboratoire de matériaux avancés et phénomènes quantiques. Université \\ de Tunis El Manar ll. Faculté des Sciences de Tunis, 2092, Tunisia \\ ${ }^{3}$ Laboratoire Interdisciplinaire Carnot de Bourgogne, UMR CNRS 6303, \\ Université de Bourgogne Franche-Comté, BP 47870, F-21078 Dijon, France
}

\begin{abstract}
We develop an inverse geometric optimization technique that allows the derivation of optimal and robust exact solutions of low-dimension quantum control problems driven by external fields: we determine in the dynamical variable space optimal trajectories constrained to robust solutions by Euler-Lagrange optimization; the control fields are then derived from the obtained robust geodesics and the inverted dynamical equations. We apply this method, referred to as robust inverse optimization (RIO), to design optimal control fields producing a complete or half population transfer and a NOT quantum gate robust with respect to the pulse inhomogeneities. The method is versatile and can be applied to numerous quantum control problems, e.g. other gates, other types of imperfections, Raman processes, or dynamical decoupling of undesirable effects.
\end{abstract}

Introduction.- Designing solutions of a system driven by external controls that are optimal with respect to practical costs such as area of the controls, energy or duration is a well known problem [1]. The necessary conditions of optimality were established by Pontryagin via a maximum principle [2]. Based on this approach, various optimal quantum problems have been solved from low- [3-6] to large-dimensional [7, 8] systems.

Solutions that additionally feature robustness has become a major issue in quantum physics, especially in quantum information processing, where ultra highfidelity solutions are required (typically with relative errors below $10^{-4}$ ) [9]. Small imperfections in the design can cause fatal deviations of the performance. Robustness can be specifically taken in to account using adiabatic [10, 11], composite [12-14], combined [15] or shortcut to adiabaticity [16-19] techniques. However, these methods are not optimal and usually cost non-necessary energy and time.

Combining robustness constraints with the optimization methods has thus become a major challenge [20]. Numerical optimal control techniques based on time discretization with thousands of parameters to be optimized [21] can lead to very different results depending on the algorithm and the initial condition used. Alternative techniques involving from a few tens [22] to a few [23] parameters to be optimized have been developed, but they do not provide global optimal solutions in principle since they are based on restricted parametrizations. A recent proposal using Pontryagin's maximum principle (PMP) in an extended Hilbert space [24] allows an elegant integration of the robustness constraints, but leads to complicated systems to solve, only tractable for very simple targets, typically complete population transfers. A geometrical approach has been shown to provide optimal single-qubit phase gates [25]. All these methods use a direct optimization procedure, i.e. with the dynamical

\footnotetext{
* sguerin@u-bourgogne.fr
}

equations as constraints, which makes complicated the simultaneous integration of the robustness constraints.

In this Letter, we propose an alternative method of optimization based on inverse engineering: We apply in a first stage the Euler-Lagrange optimization [26] constrained by robustness integrals and by the boundaries ensuring exact fidelity in the dynamical variable space without invoking the dynamical equations. The constrained Euler-Lagrange optimization leads thus to a robust and exact geodesic. In a second stage, we derive the control field parameters from the geodesic and the dynamical equation, i.e. the time-dependent Schrödinger equation for the present quantum control problem, formulated in an inverted way, in which we express the controls from the dynamical variables.

We describe the technique by applying it to determine complete and partial transfers, as well as single-qubit gates that features all the desired properties with respect to the control pulse area (chosen as the cost): exactfidelity, optimality and robustness. The general applicability of the method is finally discussed with an emphasis on specific important quantum control problems.

Inverse engineering method in the dynamical variable space.- We can consider, without loss of generality, traceless Hamiltonians $H_{\lambda}=H_{0}+\lambda V$ (in units such that $\hbar=1$ ), where

$$
H_{0}=\frac{1}{2}\left(\begin{array}{cc}
-\Delta & \Omega \\
\Omega & \Delta
\end{array}\right),
$$

models the qubit $\{|0\rangle,|1\rangle\}$ with the control parameters: the pulsed Rabi frequency $\Omega \equiv \Omega(t)$ (considered positive without loss of generality) and the detuning $\Delta \equiv \Delta(t)$, and $\lambda$ gathers unknown (time-independent) parameters representing the error in the description of the model. An error with respect to (i) the pulse amplitude (or area for a fixed pulse duration), i.e. the pulse inhomogeneities, is modeled with $V=\Omega \sigma_{x} / 2$ and $\lambda=\alpha$ corresponds to the relative deviation of the pulse amplitude; (ii) the detuning is modeled with $V=-\sigma_{z} / 2$ and $\lambda=\delta$ corresponds to the deviation of the detun- 
ing. The solution $\left|\phi_{0}(t)\right\rangle=U_{0}\left(t, t_{i}\right)\left|\phi_{0}\left(t_{i}\right)\right\rangle$ of the time dependent Schrödinger equation (TDSE) associated to the qubit without errors, $i \hbar \frac{\partial}{\partial t} U_{0}\left(t, t_{i}\right)=H_{0} U_{0}\left(t, t_{i}\right)$ with $U_{0}\left(t, t_{i}\right)$ the propagator from the initial time $t_{i}$ to time $t$, is conveniently parameterized with three angles, the mixing angle $\theta \equiv \theta(t) \in[0, \pi]$, a relative $\varphi \equiv \varphi(t) \in[-\pi, \pi]$ and a global phase $\gamma \equiv \gamma(t) \in[0,2 \pi]$, as

$$
\left|\phi_{0}(t)\right\rangle=\left(\begin{array}{c}
\cos \frac{\theta}{2} e^{i \varphi / 2} \\
\sin \frac{\theta}{2} e^{-i \varphi / 2}
\end{array}\right) e^{-i \gamma / 2}
$$

The TDSE can be equivalently rewritten as

$$
\begin{aligned}
\dot{\theta} & =\Omega \sin \varphi, \\
\dot{\varphi} & =\Delta+\Omega \cos \varphi \cot \theta, \\
\dot{\gamma} & =\Omega \frac{\cos \varphi}{\sin \theta}=\dot{\theta} \frac{\cot \varphi}{\sin \theta} .
\end{aligned}
$$

The inverse-engineering method consists in determining the Hamiltonian elements (the controls) from the dynamical variables by inverting the TDSE: $H_{0}=$ $i \hbar\left(\frac{\partial}{\partial t} U_{0}\left(t, t_{i}\right)\right) U_{0}^{\dagger}\left(t, t_{i}\right)$, i.e. from inversion of Eqs. (3):

$$
\begin{aligned}
& \Delta=\dot{\varphi}-\dot{\gamma} \cos \theta, \\
& \Omega=\sqrt{\dot{\theta}^{2}+\dot{\gamma}^{2} \sin ^{2} \theta}=|\dot{\gamma}| \sqrt{(\dot{\widetilde{\theta}})^{2}+\sin ^{2} \widetilde{\theta}}
\end{aligned}
$$

with $\dot{\tilde{\theta}} \equiv \frac{d \widetilde{\theta}}{d \gamma}$. Since the optimization cost (the pulse area) and the integrals of robustness [see Eqs. (6)] depend on $\theta(t)$ and $\gamma(t)$, we can consider them as the dynamical variables providing a geometric representation of the problem, and the third variable $\varphi(t)$ is given by $(3 \mathrm{c}), \cot \varphi=\dot{\gamma} \sin \theta / \dot{\theta}$, from which we obtain $\dot{\varphi}=$ $\left(\ddot{\theta} \dot{\gamma} \sin \theta-\ddot{\gamma} \dot{\theta} \sin \theta-\dot{\gamma} \dot{\theta}^{2} \cos \theta\right) /\left(\dot{\theta}^{2}+\dot{\gamma}^{2} \sin ^{2} \theta\right)$. In the right part of Eq. (4b), we have assumed that one can write $\theta(t)$ as a function of $\gamma(t): \widetilde{\theta}(\gamma) \equiv \theta(t)$. In general we are led to consider multiple functions $\widetilde{\theta}_{j}(\gamma)$, each depending on the time interval, in order to get the optimal solution (see for instance Fig. 3). We note that the pulse area from the initial $t_{i}$ to the final $t_{f}$ times (denoting $\gamma_{i} \equiv \gamma\left(t_{i}\right), \gamma_{f} \equiv \gamma\left(t_{f}\right)$ and assuming a monotonic $\gamma(t)$, such that $\dot{\gamma}(t)>0$ ) can be written as

$$
\mathcal{A}=\int_{t_{i}}^{t_{f}} d t \Omega(t)=\int_{\gamma_{i}}^{\gamma_{f}} d \gamma \sqrt{(\dot{\tilde{\theta}})^{2}+\sin ^{2} \widetilde{\theta}}
$$

which does not depend on the time-dependance of $\gamma(t)$, but only on $\tilde{\theta}(\gamma)$ (and its derivative). In this representation, it is thus relevant to consider trajectories $\widetilde{\theta}(\gamma)$ in the parameter space formed by the angles $\gamma, \theta$ and $\widetilde{\varphi}(\gamma) \equiv \varphi(t)$ is given by (3c) for a given trajectory. We could have considered alternatively a representation with multiple functions $\widetilde{\gamma}_{j}(\theta)$. However we will see that the chosen representation $\widetilde{\theta}(\gamma)$ is more convenient for the technical determination of the geodesics.

As a simplification of the presentation, we consider in this Letter robustness with respect to the pulse area (or identically to the pulse amplitude or inhonomegenities for a given time of interaction). It is shown below to allow one to consider the problem in the parameter space without invoking specific time parametrization: a robust optimal solution corresponds to a special trajectory $\widetilde{\theta}(\gamma)$ (the same when we consider optimization with respect to the pulse area, pulse energy or pulse duration for a given bound of its amplitude). The construction of the actual controls, i.e. the Rabi frequency $\Omega$ and the detuning $\Delta$, from (4) necessitates to use a specific time parametrization, $\gamma(t)$, which can be chosen at will for the optimization with respect to the pulse area. On the other hand, the optimization with respect to the pulse duration corresponds to a specific time parametrization [see Eq. (8)].

We denote $\left|\phi_{\lambda}(t)\right\rangle$ the state of the complete dynamics including the error, solution of the TDSE $i \hbar \frac{\partial}{\partial t}\left|\phi_{\lambda}(t)\right\rangle=$ $H_{\lambda}\left|\phi_{\lambda}(t)\right\rangle$. The single-shot shaped pulse method [17] allows one to define trajectories, in the dynamical variables space, resistant to errors. It can be formulated by a perturbative expansion of $\phi_{\lambda}\left(t_{f}\right)$ with respect to $\lambda$, $\left\langle\phi_{T} \mid \phi_{\lambda}\left(t_{f}\right)\right\rangle=1+O_{1}+O_{2}+O_{3}+\cdots$, where $O_{n}$ denotes the error term of total order $n: O_{n} \equiv O\left(\lambda^{n}\right)$ and $\left|\phi_{T}\right\rangle$ the target state. The first two terms read

$$
\begin{gathered}
O_{1}=-i \int_{t_{i}}^{t_{f}}\left\langle\phi_{0}(t)|V(t)| \phi_{0}(t)\right\rangle d t \equiv-i \int_{t_{i}}^{t_{f}} e(t) d t, \\
O_{2}=(-i)^{2} \int_{t_{i}}^{t_{f}} d t \int_{t_{i}}^{t} d t^{\prime}\left[e(t) e\left(t^{\prime}\right)+f(t) \bar{f}\left(t^{\prime}\right)\right], \\
=-\frac{1}{2}\left[\int_{t_{i}}^{t_{f}} d t e(t)\right]^{2}-\int_{t_{i}}^{t_{f}} d t f(t) \int_{t_{i}}^{t} d t^{\prime} \bar{f}\left(t^{\prime}\right),
\end{gathered}
$$

with $e=-\frac{1}{2}\left(\delta \cos \theta-\alpha \dot{\gamma} \sin ^{2} \theta\right)$, and $f=\frac{1}{2}[\delta \sin \theta+$ $\left.\alpha\left(\frac{1}{2} \dot{\gamma} \sin 2 \theta-i \dot{\theta}\right)\right] e^{i \gamma}$. The other terms can be determined from a symbolic diagram [17]. We denote at a certain order $n:\left\langle\phi_{T} \mid \phi_{\alpha, \delta}\left(t_{f}\right)\right\rangle_{n}=1+O_{1}+O_{2}+\cdots+O_{n}$. One notes the remarkable property that, assuming a function $\widetilde{\theta}(\gamma)$ and a monotonic $\gamma(t)$ (such that $\dot{\gamma}(t)>0$ ), when one considers the robustness with respect to solely $\alpha$ (i.e. $\delta=$ $0)$, then the integrals $O_{n}$ do not depend on the particular time-parametrization of $\gamma(t)$ since

$$
\begin{aligned}
& \int_{t_{i}}^{t} e(t) d t=\frac{1}{2} \alpha \int_{t_{i}}^{t} d t \dot{\gamma} \sin ^{2} \theta=\frac{1}{2} \alpha \int_{\gamma_{i}}^{\gamma} d \gamma \sin ^{2} \widetilde{\theta} \\
& \int_{t_{i}}^{t} f(t) d t=\frac{1}{2} \alpha \int_{\gamma_{i}}^{\gamma} d \gamma\left(\frac{1}{2} \sin 2 \widetilde{\theta}-i \frac{d \widetilde{\theta}}{d \gamma}\right) e^{i \gamma}
\end{aligned}
$$

The opposite situation of a decreasing $\gamma(t)(\dot{\gamma}(t)<0)$ would add a minus sign in the right hand sides of Eqs. (7).

One can thus design an optimal robust trajectory $\widetilde{\theta}(\gamma)$ when $\delta=0$. The robust optimal time $T_{\min }$ is determined from the particular time-parametrization $\gamma(t)$ of the same trajectory $\tilde{\theta}(\gamma)$ leading to a flat pulse (of chosen amplitude $\Omega_{0}$ ):

$$
T_{\min }=\frac{1}{\Omega_{0}} \int_{\gamma_{i}}^{\gamma_{f}} d \gamma \sqrt{(\dot{\widetilde{\theta}})^{2}+\sin ^{2} \widetilde{\theta}} .
$$


We notice that robustness with respect to $\delta$ could be considered, from the trajectory $\widetilde{\theta}(\gamma)$ previously derived by exploiting the time-dependence of $\gamma(t)$ (anticipated different from the one derived above for the time optimization).

The method, referred in short to as robust inverse optimization (RIO), is applied below for deriving first the optimal robust solution of two typical examples of population transfer: complete transfer and half coherent superposition (referred to as half transfer). We next consider a more complex target: the optimal robust quantum gate.

RIO for optimal robust population transfer. For the case of a population transfer to a target state $\left|\phi_{T}\right\rangle$, the final global phase is not a priori fixed and is not robust since it is irrelevant. The figure of merit up to the third order reads

$$
\left|\left\langle\phi_{T} \mid \phi_{\alpha, \delta}\left(t_{f}\right)\right\rangle\right|^{2}=1+\tilde{O}_{2}, \widetilde{O}_{2}=\left|\int_{t_{i}}^{t_{f}} f(t) d t\right|^{2} .
$$

The main result of the Letter is the following: The problem of optimal nullification up to the third order can be formulated as an optimization problem: finding the trajectory $\widetilde{\theta}(\gamma)$ that minimizes the pulse area (5)

$$
\mathcal{A}(\widetilde{\theta})=\int_{\gamma_{i}}^{\gamma_{f}} d \gamma \sqrt{(\dot{\tilde{\theta}})^{2}+\sin ^{2} \widetilde{\theta}} \equiv \int_{\gamma_{i}}^{\gamma_{f}} d \gamma \mathcal{L}_{0}(\gamma, \widetilde{\theta})
$$

under the constraints $\widetilde{O}_{2}=0$ rewritten for convenience as

$$
\begin{array}{rlr}
\psi_{1}(\widetilde{\theta}) & =-\frac{1}{4} \int_{\gamma_{i}}^{\gamma_{f}} d \gamma(\sin 2 \widetilde{\theta}-2 \widetilde{\theta}) \sin \gamma \equiv \int_{\gamma_{i}}^{\gamma_{f}} d \gamma \varphi_{1}(\gamma, \widetilde{\theta}) \\
& =-\frac{1}{2}\left(\theta_{f} \cos \gamma_{f}-\theta_{i} \cos \gamma_{i}\right), \\
\psi_{2}(\widetilde{\theta}) & =\frac{1}{4} \int_{\gamma_{i}}^{\gamma_{f}} d \gamma(\sin 2 \widetilde{\theta}-2 \widetilde{\theta}) \cos \gamma \equiv \int_{\gamma_{i}}^{\gamma_{f}} d \gamma \varphi_{2}(\gamma, \widetilde{\theta}) \\
& =\frac{1}{2}\left(\theta_{i} \sin \gamma_{i}-\theta_{f} \sin \gamma_{f}\right)
\end{array}
$$

with the initial state characterized by the angles $\left(\theta_{i} \equiv\right.$ $\left.\theta\left(t_{i}\right), \varphi_{i} \equiv \varphi\left(t_{i}\right), \gamma_{i}\right)$ and the final target state $\left(\theta_{f} \equiv\right.$ $\left.\theta\left(t_{f}\right), \varphi_{f} \equiv \varphi\left(t_{f}\right), \gamma_{f}\right)$. This can be solved by the Lagrange multiplier method extended to the function space as follows: The trajectory $\widetilde{\theta}(\gamma)$ is solution of

$$
\operatorname{grad} \mathcal{A}(\widetilde{\theta})+\lambda_{1} \operatorname{grad} \psi_{1}(\widetilde{\theta})+\lambda_{2} \operatorname{grad} \psi_{2}(\widetilde{\theta})=0,
$$

with $\lambda_{j}, j=1,2$, the Lagrangian multipliers associated to the two constraints, where the gradient is defined according to the Euler-Lagrange equation (which is zero without constraint):

$$
\operatorname{grad} \mathcal{A}(\widetilde{\theta})=\frac{\partial \mathcal{L}_{0}}{\partial \widetilde{\theta}}-\frac{d}{d \gamma}\left(\frac{\partial \mathcal{L}_{0}}{\partial \dot{\tilde{\theta}}}\right)
$$

and similarly for $\operatorname{grad} \psi_{j}(\widetilde{\theta}), j=1,2$. We obtain the differential equation

$$
\begin{aligned}
\ddot{\widetilde{\theta}} & =2(\dot{\widetilde{\theta}})^{2} \operatorname{cotan} \widetilde{\theta}+\sin \widetilde{\theta} \cos \widetilde{\theta} \\
& +\left(\lambda_{1} \sin \gamma-\lambda_{2} \cos \gamma\right)\left((\dot{\widetilde{\theta}})^{2}+\sin ^{2} \widetilde{\theta}\right)^{3 / 2}
\end{aligned}
$$

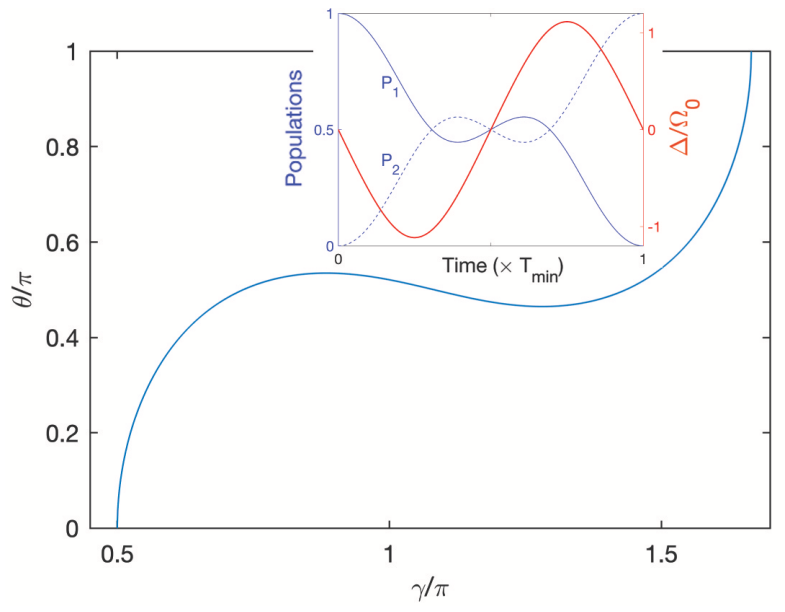

FIG. 1. Optimal robust geodesic $\widetilde{\theta}(\gamma)$ in the dynamical variable space $(\gamma, \theta)$ determined from numerical solution of (14) corresponding to $\lambda_{1} \approx-1.11505, \lambda_{2} \approx-0.30473$ (leading to $\left.\gamma_{f}=5 \pi / 3\right)$. Inset: Resulting detuning and dynamics of the populations $P_{j}, j=1,2$, for robust timeoptimal control [obtained for a flat pulse of Rabi frequency $\Omega_{0}$ according to (8)] showing the complete population transfer with the optimal time $T_{\min } \approx 5.84 / \Omega_{0}$. The detuning has the form of a complete period of the elliptic cosine $\Delta=\Delta_{0} \mathrm{cn}\left(4 K(m) t / T_{\min }+K(m), m\right), t \in\left[0, T_{\min }\right]$, with $K(m)$ the complete elliptic integral of the first kind, $m=0.235$, and $\Delta_{0}=8 K(m) \sqrt{m} / T_{\min } \approx 1.114 \Omega_{0}$.

The optimal robust trajectory $\widetilde{\theta}_{\text {opt }}(\gamma)$, solution of (14), is obtained for the set of values of $\lambda_{1}$ and $\lambda_{2}$, which satisfies (11) (and we select the trajectory of smallest pulse area in case of more than one solution). We remark that the value of $\gamma_{f}$ results from this solution.

As a first example, we consider the complete population transfer from the ground state: $\theta_{i}=0, \theta_{f}=\pi$, $\gamma_{i}=\varphi_{i}$. Equation $(3 \mathrm{c}), \cot \varphi=\dot{\tilde{\gamma}} \sin \theta=\sin \tilde{\theta} / \dot{\widetilde{\theta}}$, implies $\varphi_{i}=\varphi_{f}=\pi / 2$. We can show that $\dot{\tilde{\theta}}$ and $\ddot{\widetilde{\theta}}$ are initially and finally infinite, and that the trajectory is symmetric about $\theta=\pi / 2$ (which implies $\lambda_{1}=\lambda_{1} \sin \gamma_{f}-\lambda_{2} \cos \gamma_{f}$ ). The obtained trajectory and the corresponding driving parameters (for a flat time-optimum pulse) are shown in Fig. 1. We notice that the optimal robust trajectory $\widetilde{\theta}(\gamma)$ is a more convenient representation since it forms a function contrary to the inverse one $\widetilde{\gamma}(\theta)$. We remark that we recover the robust optimal solution that has been derived in [24] by the PMP method in an extended Hilbert space.

We next focus on a (more complex) typical example of partial population transfer: the half transfer of internal (relative) phase $\varphi_{0}$ (up to an irrelevant global phase $\left.\gamma_{0} / 2\right)$, targeted from the ground state $|0\rangle$ :

$$
\left|\phi\left(t_{f}\right)\right\rangle=\left|\phi_{T}\right\rangle \equiv \frac{1}{\sqrt{2}}\left(\begin{array}{c}
e^{i \varphi_{0} / 2} \\
e^{-i \varphi_{0} / 2}
\end{array}\right) e^{-i \gamma_{0} / 2} .
$$

This imposes the boundaries $\theta_{i}=0, \theta_{f}=\pi / 2, \gamma_{i}=\varphi_{i}=$ $\pi / 2, \varphi_{f}=\varphi_{0}$, and $\gamma_{f}=\gamma_{0}$. The obtained trajectory 


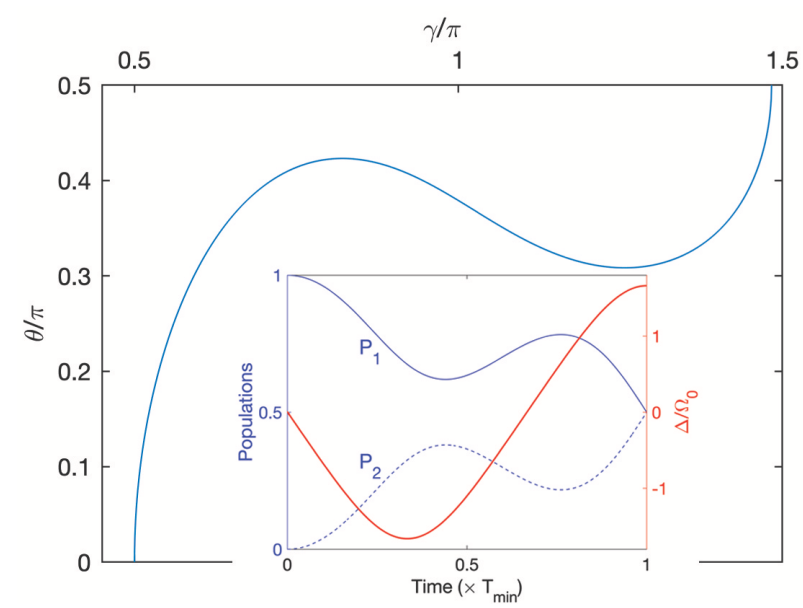

FIG. 2. Optimal robust geodesic $\tilde{\theta}(\gamma)$ corresponding to $\lambda_{1} \approx-1.69741, \lambda_{2} \approx-0.64653$ (leading to $\gamma_{f} \approx 1.48 \pi$ ). Inset: Detuning and dynamics of the populations $P_{j}, j=$ 1,2 , for robust time-optimal control (for a flat pulse of Rabi frequency $\Omega_{0}$ ) showing the half superposition. We obtain the optimal time $T_{\min } \approx 4.05 / \Omega_{0}$. The detuning has the form of three-quarters of the elliptic cosine period $\Delta=\Delta_{0} \operatorname{cn}\left(3 K(m) t / T_{\min }+K(m), m\right)$ with $m=0.4$, and $\Delta_{0}=6 K(m) \sqrt{m} / T_{\min } \approx 1.66 \Omega_{0}$.

$\widetilde{\theta}_{\text {opt }}(\gamma)$ and the corresponding driving parameters (for a flat pulse) are shown in Fig. 2. From this trajectory, we determine $\dot{\widetilde{\gamma}}_{f}=0$, which, from Eq. (3c), gives the optimal relative phase $\varphi_{0}=\pi / 2$.

RIO for robust quantum gate - Achieving a quantum gate requires the additional robust control of the global phase $\gamma$. A traceless hamiltonian can only generate the $\mathrm{SU}(2)$-type gate, which is taken as the targeted propagator: $U_{0}=\left(\begin{array}{cc}a & -b^{*} \\ b & a^{*}\end{array}\right)$ with $|a|^{2}+|b|^{2}=1$. The figure of merit usually adopted to determine the fidelity of a quantum gate is defined as $\mathcal{F}=\frac{1}{2}\left|\operatorname{Tr}\left(U_{0}^{\dagger} U\right)\right|$, where $U$ is the actual propagator. Up to the third order, it involves then the real part of the integrals $O_{1}, O_{3}$ (which are both zero), and $\mathrm{O}_{2}$ :

$$
\mathcal{F}=1+\mathcal{R}\left(O_{2}\right)=1-\frac{1}{2}\left[\int_{t_{i}}^{t_{f}} d t e(t)\right]^{2}-\frac{1}{2}\left|\int_{t_{i}}^{t_{f}} f(t) d t\right|^{2},
$$

which is one when both $\int_{t_{i}}^{t_{f}} d t e(t)=0$ and $\int_{t_{i}}^{t_{f}} f(t) d t=$ 0 . Robustness at third order can be thus expressed in this case (assuming $\dot{\gamma}(t)>0$ ) by (11) and the integral $\int_{\gamma_{i}}^{\gamma_{f}} d \gamma \sin ^{2} \widetilde{\theta}=0$. Since the argument of the latter is positive, the integral cannot be 0 and we conclude that $\gamma(t)$ cannot be monotonic. We have thus to consider two (continuous) functions

$$
\left\{\begin{array}{l}
\tilde{\theta}_{+}(\gamma) \quad \text { for } \dot{\gamma} \geq 0, \gamma=\left[\gamma_{i}, \gamma_{m}\right] \\
\tilde{\theta}_{-}(\gamma) \text { for } \dot{\gamma}<0, \gamma=\left[\gamma_{f}, \gamma_{m}[\right.
\end{array}\right.
$$

with $\theta_{m}=\tilde{\theta}_{+}\left(\gamma_{m}\right)=\tilde{\theta}_{-}\left(\gamma_{m}\right)$ and the integrals to be

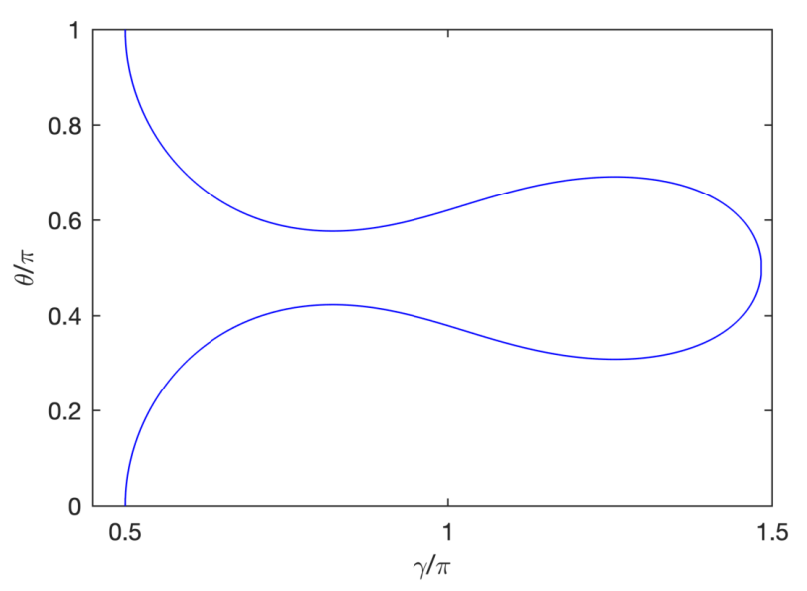

FIG. 3. Optimal robust geodesic $\widetilde{\theta}(\gamma)$ corresponding to the NOT gate. It corresponds to two consecutive symmetric trajectories of Fig. 2.

nullified become:

$$
\begin{aligned}
0 & =\int_{\gamma_{i}}^{\gamma_{m}} d \gamma \sin ^{2} \widetilde{\theta}_{+}-\int_{\gamma_{f}}^{\gamma_{m}} d \gamma \sin ^{2} \widetilde{\theta}_{-} \\
0 & =\frac{1}{2} \int_{\gamma_{i}}^{\gamma_{m}} d \gamma e^{i \gamma}\left(\sin 2 \widetilde{\theta}_{+}-2 \widetilde{\theta}_{+}\right)-i\left(\theta_{f} e^{i \gamma_{f}}-\theta_{i} e^{i \gamma_{i}}\right) \\
& -\frac{1}{2} \int_{\gamma_{f}}^{\gamma_{m}} d \gamma e^{i \gamma}\left(\sin 2 \widetilde{\theta}_{-}-2 \widetilde{\theta}_{-}\right)
\end{aligned}
$$

We consider the typical NOT-type gate: $U_{N O T}=$ $\left(\begin{array}{cc}0 & -e^{i \kappa} \\ e^{-i \kappa} & 0\end{array}\right)$, which means robust control to state $|1\rangle$ from the ground state $|0\rangle$, i.e. ideally: $\left|\phi_{\lambda}\left(t_{i}\right)\right\rangle=$ $|0\rangle,\left|\phi_{\lambda}\left(t_{f}\right)\right\rangle=\left|\phi_{T}\right\rangle \equiv e^{-i \kappa}|1\rangle$, with the phase $\kappa=\left(\varphi_{f}+\right.$ $\left.\gamma_{f}\right) / 2$, which should be additionally controlled in a robust way. This control implies the boundaries $\theta_{i}=0, \theta_{f}=$ $\pi, \gamma_{i}=\varphi_{i}=\varphi_{f}=\pi / 2$. The symmetric form $\gamma_{f}=\gamma_{i}$ (giving $\kappa=\pi / 2$ ), $\tilde{\theta}_{-}(\gamma)=2 \theta_{m}-\tilde{\theta}_{+}(\gamma), \theta_{f}=2 \theta_{m}$, for which the integral (18a) is automatically satisfied, is optimal. It leads to Eqs. (11) for the half transfer where $\gamma_{f}$ is replaced by $\gamma_{m}$. This means that the optimal robust NOT gate is achieved when two consecutive optimal robust half transfers are achieved. The resulting trajectory is shown in Fig. 3. It corresponds to a minimum time $T_{\min } \approx 8.1 / \Omega_{0}$ (for a flat pulse of Rabi frequency $\Omega_{0}$ ) and to a pulse area of $2.58 \pi$.

Discussions and conclusions. - The technique of inverse optimization we have developed allows the design of optimal and robust solutions of quantum control problems of the general form $H=H_{0}+\lambda V$, where robustness is meant with respect to $\lambda$. Its applicability necessitates the knowledge of the parametrization of the dynamics generated by $H_{0}$ and robustness is considered by perturbation of $\lambda V$. This includes low-dimensional dynamical symmetries for $H_{0}$, typically $\mathrm{SU}(2), \mathrm{SU}(3)$ [27] and $\mathrm{SU}(4)$ [28], for which dynamical invariants can be derived. But this does not limit the applicability to two-, 
three- or four-level systems; higher dimensions with specific symmetries can be considered [29]. The RIO method can treat robust optimization of the following multi-level problems:

(i) Stimulated Raman exact passage (STIREP) [18, 19] featuring a $\mathrm{SU}(2)$ symmetry in the resonant case, or more generally a $\mathrm{SU}(3)$ symmetry;

(ii) Two-qubit gate, represented as a four-level problem in its simplest form: Following Ref. [30], one can compensate the error in the phase of a two-qubit controlledPHASE gate (e.g. implemented in an ion trap) using interactions of the form $T_{1} \equiv \frac{1}{2} \sigma_{x} \otimes \sigma_{x}, T_{2} \equiv \frac{1}{2} \sigma_{x} \otimes \sigma_{y}$ and $T_{3} \equiv \frac{1}{2} \mathbb{1} \otimes \sigma_{z}$, which feature $\mathrm{SU}(2)$ symmetry;

(iii) Qudit gate (with an arbitrary dimension $d$ ), at the heart of quantum Fourier transform (a key ingredient of many quantum algorithms), in a multi-pod configuration with some overlapping controls [31] or with circulant symmetries [32].

The perturbation $\lambda V$ is not limited to imperfections of the driving pulse, but can also concern the leakeage to undesirable states [33] or to a lossy environnement (for instance leading to dephasing noise [34]), where the latter problem takes the general form $L=L_{0}+\lambda V$ via Lindbladians. The application of the method can be then interpreted as a dynamical decoupling inverse optimization. Robustness and dynamical decoupling can also be treated simultaneously.

\section{ACKNOWLEDGMENTS}

We acknowledge support from the Investissements d'Avenir programs, project ISITE-BFC /IQUINS (ANR15-IDEX-03), the EUR-EIPHI Graduate School (17EURE-0002) and from the European Union's Horizon 2020 research and innovation program under the Marie Sklodowska-Curie grant agreement No. 765075 (LIMQUET). KL acknowledges additional support from the CSC (China Scholarship Council). SG acknowledges discussions with D. Sugny.
[1] S. Glaser, U. Boscain, T. Calarco, et al. Training Schrödinger's cat: quantum optimal control, Eur. Phys. J. D 69, 279 (2015).

[2] L.S. Pontryagin, V.G. Boltyanskii, R.V. Gamkrelidze, E.F. Mischenko, The Mathematical Theory of Optimal Processes (Wiley, New York, 1962).

[3] N. Khaneja, R. Brockett, and S. J. Glaser, Phys. Rev. A 63, 032308 (2001).

[4] U. Boscain, G. Charlot, J.-P. Gauthier, S. Guérin, and H. R. Jauslin, J. Math. Phys. 43, 5 (2002).

[5] D. D'Alessandro, Introduction to Quantum Control and Dynamics (Chapman and Hall, Boca Raton, FL, 2008).

[6] B. Bonnard, D. Sugny, Optimal Control with Applications in Space and Quantum Dynamics, AIMS on Applied Mathematics (American Institute of Mathematical Sciences, Springfield, 2012), Vol. 5.

[7] S. Rice and M. Zhao, Optimal Control of Molecular Dynamics (Wiley, New York, 2003).

[8] R.C.C. Brif and H. Rabitz, New J. Phys. 12, 075008 (2010).

[9] M. A. Nielsen and I. L. Chuang, Quantum Computation and Quantum Information (Cambridge University Press, Cambridge, U.K., 2000).

[10] N. V. Vitanov, A. A. Rangelov, B. W. Shore, and K. Bergmann, Rev. Mod. Phys. 89, 015006 (2017).

[11] G. Dridi, S. Guérin, V. Hakobyan, H. R. Jauslin, and H. Eleuch, Phys. Rev. A 80, 043408 (2009).

[12] S. Wimperis, J. Magn. Reson. 109, 221 (1994).

[13] J.A. Jones, Phys. Rev. A 87, 052317 (2013).

[14] G. T. Genov, D. Schraft, T. Halfmann, and N.V. Vitanov, Phys. Rev. Lett. 113, 043001 (2014).

[15] B. T. Torosov, S. Guérin, and N. V. Vitanov, Phys. Rev. Lett. 106, 233001 (2011).

[16] D. Guéry-Odelin, A. Ruschhaupt, A. Kiely, E. Torrontegui, S. Martínez-Garaot, J. G. Muga, Rev. Mod. Phys. 91, 045001 (2019). Adv. At. Mol. Opt. Phys. 62, 117 (2013).

[17] D. Daems, A. Ruschhaupt, D. Sugny, and S. Guérin,
Phys. Rev. Lett. 111, 050404 (2013).

[18] V. Dorier, M. Gevorgyan, A. Ishkhanyan, C. Leroy, H. R. Jauslin, and S. Guérin, Phys. Rev. Lett. 119, 243902 (2017).

[19] X. Laforgue, Xi Chen, and S. Guérin, Phys. Rev. A. 100, 023415 (2019).

[20] S. J. Glaser, U. Boscain, T. Calarco, C. Koch,W. Kockenberger, R. Kosloff, I. Kuprov, B. Luy, S. Schirmer, T. Schulte-Herbrüggen, D. Sugny, and F. Wilhelm, Eur. Phys. J. D 69, 279 (2015).

[21] N. Khaneja, T. Reiss, C. Kehlet, T. Schulte-Herbrüggen, and S.J. Glaser, J. Magn. Reson. 172, 296 (2005).

[22] T. Nöbauer, A. Angerer, B. Bartels, M. Trupke, S. Rotter, J. Schmiedmayer, F. Mintert, and J. Majer, Phys Rev. Lett. 115, 190801 (2015).

[23] L. Van-Damme, D. Schraft, G.T. Genov, D. Sugny, T. Halfmann, and S. Guérin, Phys. Rev. A 96, 022309 (2017).

[24] L. Van Damme, Q. Ansel, S.J. Glaser, and D. Sugny, Phys. Rev. A 95, 063403 (2017).

[25] J. Zeng and E. Barnes, Phys. Rev. A 98, 012301 (2018).

[26] D.P. Bertsekas, Constrained Optimization and Lagrange Multiplier Methods (Athena Scientific, Belmont , Massachusetts, 1996).

[27] E. Torrontegui, S. Martínez-Garaot, and J.G. Muga, Phys. Rev. A 89, 043408 (2014).

[28] Utkan Güngördü, Y. Wan, M.A. Fasihi, and M. Nakahara Phys. Rev. A 86, 062312 (2012).

[29] F.T. Hioe J. Opt. Soc. Am. B 4, 1327 (1987).

[30] S.S. Ivanov and N.V. Vitanov, Phys. Rev. A 92, 022333 (2015).

[31] B. Rousseaux, S. Guérin, and N.V. Vitanov, Phys. Rev. A 87, 032328 (2013).

[32] P.A. Ivanov, N.V. Vitanov, Sci Rep 10, 5030 (2020).

[33] F. Motzoi, J.M. Gambetta, P. Rebentrost, F.K. Wilhelm, Phys. Rev. Lett. 103, 110501 (2009).

[34] X. Lacour, S. Guérin, L.P. Yatsenko, N.V. Vitanov, H.R. Jauslin, Physical Review A 75, 033417 (2007). 